\title{
Correlación entre la resistencia a la insulina y T3 en adolescentes con factores de riesgo para el desarrollo de diabetes
}

Correlation between insulin resistance and T3 in adolescents with risk factors for developing diabetes

Socorro Camarillo-Romero, Eneida Camarillo-Romero², Hugo Medieta-Zeron³ ${ }^{2}$ José de Jesús Garduño-García², G. Gerardo Huitrón-Bravo $\mathbf{M}^{2}$

\section{Resumen}

Objetivo. Establecer la correlación entre las concentraciones séricas de triyodotironina y la resistencia a la insulina a través del índice HOMA en un grupo de hombres adolescentes con factores de riesgo para el desarrollo de diabetes. Materiales y métodos: estudio descriptivo de corte transversal. Participaron adolescentes con algún factor de riesgo para diabetes. Se determinó la presencia de síndrome metabólico empleando los criterios de ATPIII modificada por Cook. Se determinaron T3, T4, TSH e insulina basal. Se calculó el índice HOMA para resistencia a la insulina. Resultados. Se estudiaron 36 hombres con una edad promedio de $15.97 \pm 0.9$ años. En la población total existió correlación positiva entre T3 y HOMA-IR ( $\mathrm{r}=0.380 \mathrm{p}=0.022)$, cuando se analizó en el grupo de SM la correlación se incrementó entre T3 y HOMA-IR ( $\mathrm{r}=0.647$ p=0.031). Determinando que existe correlación positiva entre los índices de resistencia a la insulina y las concentraciones de hormona tiroidea.

Palabras Clave: resistencia a la insulina, Síndrome metabólico, Adolescentes, Triyodotironina.

\begin{abstract}
Objective: To stablish the correlation between serum triiodothyronine concentrations and insulin resistance by HOMA index in a group of adolescent men with risk factors for developing diabetes. Material and Methods. It's a descriptive cross-sectional study. The paricipants were adolescents with some risk factor for diabetes. The presence of metabolic syndrome using the modified ATPIII criteria modified by Cook. T3, T4, TSH and basal insulin were determined in all subjets. HOMA index was calculated for insulin resistance. Results. 36 men with an average age of $15.97 \pm 0.9$ years were studied. There was positive correlation between T3 and HOMA-IR $(r=0.380 \mathrm{p}=0.022)$, in the full population, when analyzing only the group of metabolic syndrome, the correlation increased $(r=0.647 \mathrm{p}=0.031)$. We confirm the existence of a positive correlation between the HOMA index and the T3 concentration.
\end{abstract}

Key words: insulin Resistance, Metabolic Syndrome, Adolecents, Triiodothyronine.

\footnotetext{
Cuerpo Académico Investigación Biomédica. Centro de Investigación en Ciencias Médicas (CICMED), Universidad Autónoma del Estado de México (UAEMéx) Cuerpo Académico Salud del Universitario. CICMED, UAEMéx.

3. Director de la Asociación Científica Latina A.C. (ASCILA), CICMED, UAEMéx.

Correspondencia: sococamarillo@yahoo.es

Recibido: 02/04/2015 Aceptado: 18/06/2015
} 


\section{Introducción}

Las alteraciones tiroideas se han relacionado con obesidad, y recientemente con resistencia a la insulina (RI). En México la prevalencia de sobrepeso/ obesidad en hombres de 12 a 19 ańos fue de 34.1\% (1). Se han realizado investigaciones en las cuales se asociaron niveles de T4 normal, T3 aumentada y TSH levemente incrementada, con el índice de masa corporal en niños aparentemente sanos (eutiroideos) (2). Otros investigadores han encontrado que los niveles T3 se asociaron con resistencia a la insulina y síndrome metabólico (3). En otros grupos han estudiado la relación entre el perfil tiroideo con la resistencia a la insulina en donde han encontrado una relación lineal moderada entre T3 y HOMA-IR (4). Los adolescentes que presentan algún factor de riesgo para diabetes tienen mayor probabilidad de presentar SM. De la misma forma, se encontró que las alteraciones endocrinas pueden asociarse con RI, e incrementarse en presencia de SM.

El presente trabajo buscó establecer la correlación entre las concentraciones séricas de triyodotironina y la resistencia a la insulina a través del índice HOMA en un grupo de adolescentes con factores para el desarrollo de diabetes.

\section{Materiales y métodos}

Se planteó un estudio transversal que constó de dos etapas. En la primera etapa se aplicó un cuestionario sobre factores de riesgo, hábitos nutricionales, se tomaron mediciones antropométricas (peso, talla, cintura, tensión arterial, composición corporal). En la segunda etapa se midieron los parámetros bioquímicos (glucosa, insulina, perfil tiroideo). Aceptando un riesgo alfa de 0.05 y un riesgo beta de 0.2 para un análisis a dos colas, se precisan 100 sujetos con antecedentes heredofamiliares de diabetes en primer grado, estimando una tasa de pérdidas de seguimento del $20 \%$.

Se invitaron a los alumnos del Nivel Medio Superior de la Universidad Autónoma del Estado de
México. Se aplicó un cuestionario, se seleccionaron a los individuos que cumplían los criterios establecidos.

Se evaluaron los siguientes parámetros. Una historia médica en la cual se les preguntó sobre los antecedentes familiares de diabetes, obesidad y enfermedades crónico degenerativas. Se les realizaron mediciones antropométricas de peso y talla para calcular el índice de masa corporal, perímetro de cintura y presión arterial. Se tomaron muestras sanguíneas en condiciones basales de ayuno de 12 horas, en las cuales se determinaron las concentraciones séricas de glucosa, insulina, perfil tiroideo. Se determinó la concentración de glucosa e insulina en cada muestra según los métodos de glucosa-oxidasa y electroinmuquimioluminiscencia. Se calculó resistencia a la insulina mediante el índice de HOMA. Los componentes del síndrome metabólico se definieron de acuerdo con la adaptación de los criterios del Cook modificado para adolescentes del National Cholesterol Education Program, Adult Treatment Panel III (NCEP / ATP-III) (5), obesidad abdominal (circunferencia de la cintura $\geq$ percentil 90), hipertrigliceridemia (triglicéridos $\geq 100 \mathrm{mg} / \mathrm{dL}$ ), niveles bajos de colesterol HDL (colesterol HDL $\leq 40 \mathrm{mg} / \mathrm{dL}$ ), hipertensión arterial (presión arterial $\geq$ percentil 90), y la glucosa en ayunas anormal (glucosa en sangre $\geq 100 \mathrm{mg} / \mathrm{dL}$ ).

La base de datos fue validada. El análisis descriptivo se realizó empleando promedios y desviaciones estándar para las variables continuas. Las prevalencias están expresadas en forma de porcentajes. Se realizó diferencia entre dos grupos en variables continuas empleando t de Student. El análisis de las variables cuantitativas continúas de exposición y resultado se llevó a cabo mediante correlación de Pearson.

El estudio se lleva a cabo siguiendo las normas deontológicas reconocidas por la Declaración de Helsinki (2009). Ley General de Salud (2010). 
Firma del consentimiento informado del padre o tutor y asentimiento informado del alumno. Cadena de custodia para el manejo y almacenamiento de muestras.

\section{Resultados}

En la primera etapa del estudio se obtuvo una participación de 981 alumnos de nivel medio superior con rango de edad de 14 a 18 ańos, el 35.4\% (296) hombres. Como resultado de la historia clínica y de las mediciones antropométricas se encontró a 158 (16.1\%) adolescentes que presentaron antecedentes heredofamiliares para diabetes en primer grado, 36 (3.7\%) tuvieron obesidad y 119 sobrepeso (12.1\%).

Para la segunda etapa de la investigación, se invitaron a 100 participantes que cubrieran los criterios de selección. De los cuales 36 fueron del sexo masculino de edades entre 14 y 18 años, las mujeres (64) tenían edades entre 15 y 18 años. En la Tabla 1 podemos observar según el criterio de selección la distribución que presentó la muestra.
Tabla 1. Distribución por sexo y criterio de selección.

\begin{tabular}{lccc} 
Sexo & $\begin{array}{c}\text { AHF 1er } \\
\text { Grado }\end{array}$ & $\begin{array}{c}\text { Sobrepeso/ } \\
\text { Obesidad }\end{array}$ & Total \\
Mujer & 45 & 19 & 64 \\
Hombre & 17 & 19 & 36 \\
Total & 62 & 19 & 100 \\
\hline
\end{tabular}

Fuente: autores

Los resultados de las pruebas bioquímicas y hormonales obtenidas se observan en la Tabla 2 , en donde se encontraron diferencias estadísticamente significativas en la resistencia a la insulina en los hombres con y sin síndrome metabólico.

En análisis correlacional se muestra en la tabla 3. En ella podemos observar como existe una correlación entre T3 con la resistencia a la insulina, misma que se duplica cuando se trata de jóvenes con síndrome metabólico.

Tabla 2. Características de estudio.

\begin{tabular}{|c|c|c|c|c|}
\hline Variable & Grupo (36) & Con SM (11) & Sin SM (25) & $\mathbf{p}$ \\
\hline Gluc Basal & $92.7(7.7)$ & $95.3(8.0)$ & $91.5(7.5)$ & 0.184 \\
\hline Insulina Basal & $14.2(8.1)$ & $20.7(9.5)$ & $11.2(5.6)$ & 0.008 \\
\hline HOMA-IR & $2.9(1.8)$ & $4.5(2.3)$ & $2.3(1.1)$ & 0.009 \\
\hline T3 & $1.27(0.25)$ & $1.37(0.17)$ & $1.23(0.27)$ & 0.065 \\
\hline T4 & $8.10(1.4)$ & 7.93(1.10) & $8.18(1.49)$ & 0.587 \\
\hline TSH & $2.38(0.96$ & $2.50(0.94)$ & $2.33(0.99)$ & 0.638 \\
\hline
\end{tabular}


Media (DE), $\mathrm{p}<0.05 \mathrm{t}$ Student

Tabla 3. Correlación de variables con T3.

\begin{tabular}{|c|c|c|c|c|c|c|}
\hline Variable & Grupo n=36 & $\mathbf{p}$ & Con SM n=11 & $\mathbf{p}$ & $\operatorname{Sin} \mathbf{S M} \mathbf{n}=\mathbf{2 5}$ & $\mathbf{p}$ \\
\hline Gluc Basal & 0.367 & 0.027 & 0.398 & 0.226 & 0.319 & 0.120 \\
\hline Insulina Basal & 0.344 & 0.040 & 0.398 & 0.030 & 0.111 & 0.597 \\
\hline HOMA-IR & 0.380 & 0.022 & 0.647 & 0.031 & 0.176 & 0.400 \\
\hline
\end{tabular}

Correlación de Pearson $\mathrm{p}<0.05$.

\section{Discusión}

El sobrepeso, la obesidad, el sedentarismo y los malos hábitos alimenticios son factores que determinan el desarrollo de la resistencia a la insulina, también llamado prediabetes. Más tarde puede evolucionar a hiperinsulinismo por la sobreproducción pancreática de insulina, llevando al organismo al desarrollo de Diabetes mellitus tipo 2, así como alteraciones en el metabolismo de los lípidos que constituyen a un factor de riesgo para el desarrollo de enfermedades cardiovasculares. El diagnóstico precoz de estos trastornos es necesario para establecer medidas preventivas que interrumpan dicha evolución y prevenir la aparición de la diabetes y de sus complicaciones.

El análisis para este estudio se realizó en el grupo de hombres considerando los cambios hormonales que presentan las mujeres a esta edad, en donde se determinó la prevalencia de síndrome metabólico encontrando $30.6 \%$, debemos tener en cuenta que los cambios subclínicos en el metabolismo de la glucosa comienzan desde la adolescencia (6), relacionados con factores predisponentes y ambientales que se presentan en síndrome metabólico. En estudios previos realizados por nuestro grupo de investigación, hemos encontrado una prevalencia de síndrome metabólico en este grupo de edad varía entre 18.6 a 35.4\% (78). Se determinó el índice HOMA, empleando el punto de corte de Keskin (9), encontrando 33\% de resistencia a la insulina en nuestros jóvenes que presentan factores de riesgo para desarrollar enfermedades crónicas degenerativas.

La población mexicana es considerada de alto riesgo para el desarrollo de diabetes y prediabetes, estando implicados varios procesos patogénicos. Por otra parte, la disfunción tiroidea ha sido asociada a diabetes y resistencia a la insulina aunque los mecanismos fisiopatológicos (10-11) involucrados no se conocen a detalle, siendo un problema multifactorial. Los procesos moleculares que interaccionan a diferentes niveles llegan a impactar en los mecanismos moleculares de la resistencia a la acción de la insulina (12-13), favoreciendo el desarrollo de la diabetes, además, la predisposición genética así como la obesidad son de los principales factores de riesgo para desarrollar alteraciones en el metabolismo de la glucosa (14). Existen estudios que han demostrado que los familiares de las personas que tienen diabetes pueden tener cambios subclínicos en estadios tempranos. Tenemos entonces que la identificación temprana de alteraciones en el metabolismo de la glucosa en adolescentes podría brindar la oportunidad para estrategias terapéuticas tempranas.

Los adolescentes que presentan algún factor de riesgo para diabetes tienen mayor probabilidad de presentar SM. Así como se encontró que las alteraciones endocrinas pueden asociarse con RI, e incrementarse en presencia de SM. 


\section{Referencias}

1. ENSANUT 2012. Encuesta nacional de salud y nutricion. 2012. INSP. México.

2. Raman K. Marwaha, Nikhil Tandon, M. K. Garg, Mohd Ashraf Ganie, A. Narang, Neena Mehan, Kuntal. Impact of Body Mass Index on Thyroid Functions in Indian Children. Clin Endocrinol. 2013;79(3):424-428.

3. Tarcin O, Abanonu GB, Yazici D, Tarcin O. Association of metabolic syndrome parameters with TT3 and FT3/FT4 ratio in obese Turkish population. Metab Syndr Relat Disord. 2012 Apr;10(2):137-42. doi: 10.1089/met.2011.0098. Epub 2012 Jan 9.

4. Rezzonico J, Niepomniszcze H, Rezzonico M, Pusiol E, Alberto M, Brenta G. Thyroid. The association of insulin resistance with subclinical thyrotoxicosis. 2011 Sep;21(9):9459. doi: 10.1089/thy.2010.0402. Epub 2011 Aug 11.

5. Cook S, Weitzman M, Auinger P, Nguyen M, Dietz WH. Prevalence of a metabolic syndrome phenotype in adolescents: findings from the third National Health and Nutrition Examination Survey, 1988-1994. Archives of pediatrics \& adolescent medicine. 2003;157(8):821-7. Epub 2003/08/13Dessein PH, Joffe BI, Stanwix AE. Subclinical hypothyroidism is associated with insulin resistance in rheumatoid arthritis. Thyroid. 2004 Jun;14(6):443-6.

6. American Diabetes Association. Type 2 diabetes in children and adolescents. Diabetes Care. 2000;23(3):381-389.

7. Camarillo-Romero Eneida, Domínguez García Ma Victoria, Amaya-Chávez Araceli, Huitrón-Bravo Gerardo, Majluf-Cruz Abraham. Dificultades en la clasificación del síndrome metabólico: El ejemplo de los adolescentes en México. Salud Pub Méx. 2010; 52(6): 524-527.
8. Halley-Castillo E, Borges G, Talavera JO, Orozco R, Vargas-Alemán $\mathrm{C}$, Huitrón Bravo G, et al. Body mass index and the prevalence of metabolic syndrome among children and adolescents in two Mexican populations. J Adolesc Health 2007;40(6):521-526

9. Keskin M, Kurtoglu S, Kendirci M, Atabek ME, Yazici C. Homeostasis Model Assessment Is More Reliable Than the Fasting Glucose/Insulin Ratio and Quantitative Insulin Sensitivity Check Index for Assessing Insulin Resistance Among Obese Children and Adolescents. Peditrics. 2005; 115(4): 500-503.

10. Xu M, Bi Y, Cui B, Hong J, Wang W, Ning G. The new perspectives on genetic studies of type 2 diabetes and thyroid diseases. Curr Genomics. 2013 Mar;14(1):33-48.

11. Dessein PH, Joffe BI and Stanwix AE. Subclinical Hypothyroidism is Associated with Insulin Resistance in Rheumatoid Arthritis. Thyroid. 2004, 14(6): 443-446.

12. Ferré N, Feliu A, García-Heredia A, Marsillach J, París N, Zaragoza-Jordana M, Mackness B, Mackness M, Escribano J, ClosaMonasterolo R, Joven J, Camps J. Impaired paraoxonase-1 status in obese children. Relationships with insulin resistance and metabolic syndrome. Clin Biochem. 2013 Sep 10.

13. Vatner DF, Weismann D, Beddow SA, Kumashiro N, Erion DM, Liao XH, Grover GJ, Webb P, Phillips KJ, Weiss RE, Bogan JS, Baxter J, Shulman GI, Samuel VT. Thyroid hormone receptor- $\mathbb{\Delta}$ agonists prevent hepatic steatosis in fat-fed rats but impair insulin sensitivity via discrete pathways. Am J Physiol Endocrinol Metab. 2013 Jul 1;305(1):E89-100

14. Canani LH, Capp C, Dora JM, Meyer EL, Wagner MS, Harney JW, Larsen PR, Gross JL, Bianco AC, Maia AL. The Type 2 Deiodinase A/G (Thr92Ala) Polymorphism Is Associated with Decreased Enzyme Velocity and Increased Insulin Resistance in Patients with Type 2 Diabetes Mellitus. J Clin Endocrinol Metab. 2005 Jun;90(6):3472-3478. 